groups in any of the included variables. Most patients reported mild pain (NRS median 3) and moderate disability. Thirty-six had additional affection of interphalangeal joints. At follow-up, the control group showed no improvements, while the intervention group showed significant improvement in all pain and functional measures, except flexion deficit. When adjusting for baseline value and time to follow-up, there were significant differences between the two groups at follow-up for pain at rest, pain following grip, self-reported function (MAP-Hand and Quick$\mathrm{DASH}$ ) and grip strength, with the intervention group showing greater improvement $(p<0.01)$

Conclusion: Occupational therapy had significant positive short-term effects on pain and function in patients referred to surgical consultation for $\mathrm{CMC} 1 \mathrm{OA}$. Disclosure of Interests: None declared DOI: 10.1136/annrheumdis-2019-eular.5051

\section{OP0162-HPR THE NEED FOR PERSONALIZED, NON- PHARMACOLOGICAL INTERVENTION PROGRAMMES IN AUTOIMMUNE CONNECTIVE TISSUE DISORDERS: RESULTS OF A EULAR-FUNDED SCOPING REVIEW WITH A NESTED, DESCRIPTIVE META-ANALYSIS}

Valentin Ritschl ${ }^{1}$, Ricardo Ferreira ${ }^{2}$, Rúben Fernandes ${ }^{2}$, Eduardo Santos ${ }^{2}$, Kim Fligelstone ${ }^{3}$, Helena Gaspar ${ }^{4}$, Linda Schraven ${ }^{5}$, Judy Ammerlaan ${ }^{6}$, Georg Stummvoll ${ }^{1}$, Maria Joao Salvador ${ }^{2}$, Janet L Poole ${ }^{7}$, Cornelia van den Ende ${ }^{8}$ Carina Boström', Tanja Stamm' ${ }^{1}$ Medical University of Vienna, Vienna, Austria; ${ }^{2}$ Centro Hospitalar e Universitário de Coimbra, Coimbra, Portugal; ${ }^{3}$ Federation of European Scleroderma Associations, London, United Kingdom; ${ }^{4}$ Portuguese League Against Rheumatic Diseases, Lisboa, Portugal; ${ }^{5}$ Federation of European Scleroderma Associations, Tournai, Belgium; ${ }^{6}$ University Medical Centre, Utrecht, Netherlands; ${ }^{7}$ University of New Mexico, Albuquerque, United States of America; ${ }^{8}$ Sint Maartenskliniek, Ubbergen, Netherlands; ${ }^{9}$ Karolinska Institutet, Stockholm, Sweden

Background: Autoimmune Connective Tissue Disorders (CTDs), including Mixed Connective Tissue Disease (MCTD), systemic sclerosis (SSc) and systemic lupus erythematous (SLE) can lead to Raynaud phenomenon, involvement of internal organs, pain in joints, skin abnormalities and muscle weakness. Consequently, people with CTDs experience limitations in activities of daily life and restrictions in participation. Programmes promoting patient education, self-management, physical activity, and healthy life style, provided by health professionals are therefore essential to these patients. However, there is a substantial gap of knowledge about the content which should be included in non-pharmacological intervention programmes.

Objectives: To identify, map and appraise the content of existent face-to-face, digital and/or distance-learning non-pharmacological intervention programmes for patients with MCTD, SSc and/or SLE to inform a EULAR funded project with the overall aim to develop a new distance learning programme realizable with digital technologies.

Methods: A scoping literature review with a nested descriptive meta-analysis was performed in MEDLINE [Pubmed], EMBASE [OVID], CINAHL [EBSCO], PsyCINFO [ProQUEST], the Cochrane Database of Systematic Reviews, OTSeeker, PEDro, and SciELO in September 2018. Furthermore, an international task-force identified additional grey literature and ongoing research projects. Eligibility checks of the articles based on pre-defined in- and exclusion criteria and data extractions were done independently by two researchers. The interventions contained in these programmes were extracted using the "Better reporting of interventions: template for intervention description and replication (TIDieR) checklist and guide". A Sankey diagram and descriptive statistics were used to illustrate the relationships between the interventions and other characteristics of the studies. The protocol was pre-published on ResearchGate.

Results: Of 6667 identified records, 94 papers were eligible. Sixty-one studies $(65 \%)$ were conducted between 2000 and 2018, mainly in the USA (35 [37\%]), Brazil (11 [12\%]) and Italy (10 [11\%]). Thirty-three studies (35\%) were randomised controlled trials, 17 (18\%) were one-group pre-post-test designs and $12(16 \%)$ clinical trials without randomisation (Figure 1). Overall, 4274 patients participated 2955 had SLE [69\%] and 1319 had SSc [31\%], thus no studies with MCTD where found. The main content of the intervention programmes included exercises (22 [23\%]), self-management (9 [10\%]) and education (7 [7\%]), with treatment goals focusing mainly on physical function (27 [29\%]), quality of life $(19[20 \%])$ and fatigue (12 [16\%]). The interventions $(67[72 \%])$ included or were based on an initial face-to-face component.
Figure 1. Sankey diagram. This diagram illustrates the relationships between the different study characteristics. The width of the grey connections lines between the bars are reflecting a higher

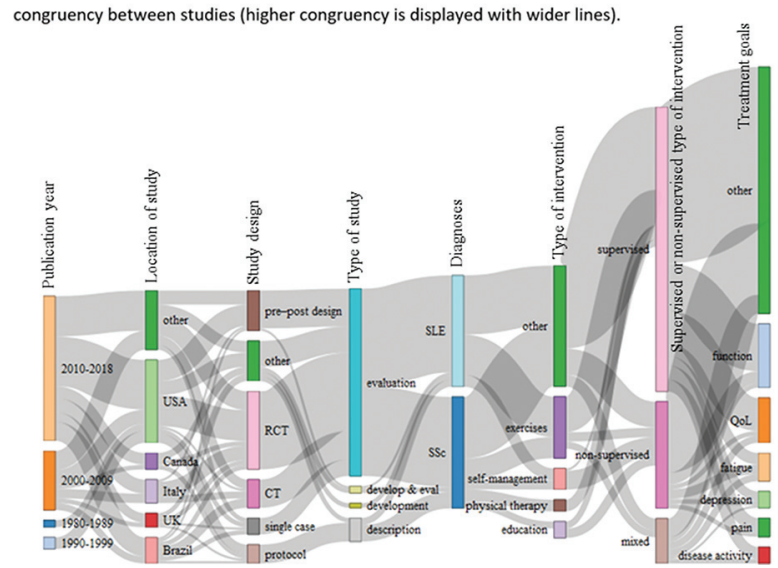

Conclusion: There was a variety of programmes content and interventions due to different consequences regarding activity limitations and participation restric tions in autoimmune CTDs. This highlights the need for personalized, multicomponent, non-pharmacological interventions.

Disclosure of Interests: Valentin Ritschl: None declared, Ricardo Ferreira: None declared, Rúben Fernandes: None declared, Eduardo Santos: None declared, Kim Fligelstone: None declared, Helena Gaspar: None declared, Linda Schraven: None declared, Judy Ammerlaan: None declared, Georg Stummvoll: None declared, Maria Joao Salvador: None declared, Janet L Poole: None declared, Cornelia van den Ende: None declared, Carina Boström: None declared, Tanja Stamm Grant/research support from: TS has received grant sup port from AbbVie., Paid instructor for: TS has received speaker fees from AbbVie, Janssen, MSD, Novartis, and Roche.

DOI: 10.1136/annrheumdis-2019-eular.896

\section{THURSDAY, 13 JUNE 2019 Other orphan diseases}

\section{OP0163 LONG-TERM EFFECTIVENESS OF CANAKINUMAB IN PATIENTS WITH CRYOPYRIN-ASSOCIATED PERIODIC SYNDROME}

J. B. Kuemmerle-Deschner ${ }^{1}$, Norbert Blank ${ }^{2}$, Michael Borte $^{3}$, Ivan Foeldvari ${ }^{4}$ Gerd Horneff ${ }^{5}$, Prasad Oommen ${ }^{6}$, Catharina Schuetz ${ }^{7}$, Frank Weller-Heinemann ${ }^{8}$, Julia Weber-Arden ${ }^{9}$, Tilmann Kallinich ${ }^{10} .{ }^{1}$ University Hospital, Tübingen, Germany; ${ }^{2}$ University Hospital, Heidelberg, Germany; ${ }^{3}$ Hospital St. Georg, Leipzig, Germany; ${ }^{4}$ Center Ped Rheumatology, Hamburg, Germany; ${ }^{5}$ Asklepios Clinic, Sankt Augustin, Germany; ${ }^{6}$ University Hospital, Düsseldorf, Germany, ${ }^{7}$ University Hospital, Dresden, Germany; ${ }^{8}$ Prof. Hess Klinik, Bremen, Germany, ${ }^{9}$ Novartis, Nuremberg, Germany; ${ }^{10}$ Charité, Berlin, Germany

Background: Treatment options for autoinflammatory diseases include antiinterleukin (IL)-1 therapies since the IL-1 pathway is crucial in immune dysregulation in patients with monogenic periodic fever syndromes.

Objectives: To gain further insights from routine clinical practice with respect to long-term effectiveness and safety of canakinumab (CAN) in pediatric and adult patients with CAPS (cryopyrin-associated periodic syndrome, including MuckleWells syndrome [MWS], familial cold autoinflammatory syndrome [FCAS], neonatal onset multisystem inflammatory disease [NOMID]/chronic infantile neurological cutaneous and articular syndrome [CINCA]), FMF (familial Mediterranean fever), TRAPS (tumor necrosis factor receptor-associated periodic syndrome) and HIDS/MKD (hyperimmunoglobulinemia D syndrome/mevalonate kinase deficiency).

Methods: RELIANCE is a prospective, non-interventional, multi-center, observational study based in Germany with a 3-year follow-up enrolling patients aged $>2$ years with clinically confirmed diagnoses who routinely received CAN. 6-monthly clinical assessment and evaluation of patient-reported outcomes is aligned with routine clinic visits. CAPS disease activity was determined by physicians' and patients' assessment. Endpoints were effectiveness and safety of CAN under standard clinical practice conditions. 